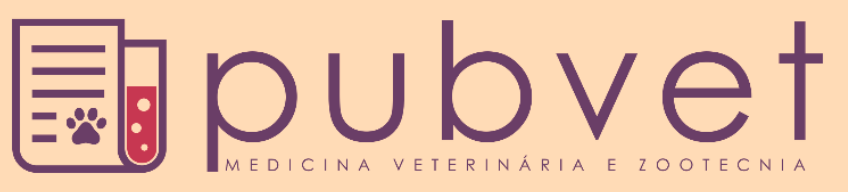

https://doi.org/10.31533/pubvet.v15n03a779.1-8

\title{
Leishmaniose visceral canina: Revisão
}

\author{
José Duarte da Fonseca Júnior ${ }^{1}{ }^{\ominus}$, Cristiane Lopes Mazzinghy ${ }^{2 *} \bullet$, Erycka Carolina França ${ }^{1}{ }^{\ominus}$, \\ Ana Clara Silva Pinow $^{4}{ }^{\circ}$, Katyane de Sousa Almeida ${ }^{5}$ \\ ${ }^{I}$ Médico veterinário. Palmas - TO Brasil. \\ ${ }^{2}$ Professora do Centro Universitário Luterano de Palmas, Departamento de Medicina Veterinária. Palmas - TO Brasil. \\ ${ }^{4}$ Graduando em Medicina Veterinária do Centro Universitário Luterano de Palmas. Palmas - TO Brasil. \\ ${ }^{5}$ Professora da Fundação Universidade Federal do Tocantins. Departamento de Medicina Veterinária e Zootecnia. Araguaína-TO Brasil. \\ *Autor para correspondência, E-mail: crislp03@yahoo.com.br
}

Resumo. A leishmaniose canina visceral é uma zoonose que possui grande repercussão na saúde animal e humana, cujo agente etiológico são os protozoários do gênero Leishmania sp. O objetivo do estudo consiste em realizar uma revisão sobre os aspectos importantes da doença, como agente etiológico, transmissão e patogenia, sinais clínicos, diagnóstico, tratamento, prevenção e controle, para acrescentar informações no âmbito da clínica médica de pequenos animais.

Palavras-chave: Hemoparasitoses, miltefosina

\section{Canine visceral leishmaniasis: Review}

Abstract. Visceral canine leishmaniasis is a zoonosis that has great repercussions on animal and human health, whose etiologic agents are the protozoa of the genus Leishmania $\mathrm{sp}$. The objective of the study is to carry out a review on the important aspects of the disease, such as etiologic agent, transmission and pathogenesis, clinical signs, diagnosis, treatment, prevention and control, to add information in the context of small animal medical clinic.

Keywords: Hemoparasitosis, miltefosine

\section{Leishmaniasis visceral canina: Revisión}

Resumen. La leishmaniasis visceral canina es una zoonosis de gran repercusión en la salud animal y humana, cuyos agentes etiológicos son los protozoos del género Leishmania sp. El objetivo del estudio es realizar una revisión sobre los aspectos importantes de la enfermedad, como agente etiológico, transmisión y patogenia, signos clínicos, diagnóstico, tratamiento, prevención y control, para agregar información en el contexto de la clínica médica de pequeños animales.

Palabras clave: Homoparasitosis, miltefosina

\section{Introdução}

Os caninos são os animais domésticos que apresentam maior proximidade com o homem devido ao estreitamento da relação homem-animal que foi estabelecida e desenvolvida com o decorrer de muitos anos (Caldart et al., 2015; Hoffmann et al., 2012). Esta relação trouxe diversos benefícios para a sociedade, dentre eles a diminuição do estresse e a melhora na capacidade de comunicação de pessoas portadoras de enfermidades graves (McNicholas et al., 2005). Por outro lado, também resultaram em consequências negativas, como o surgimento de doenças com grande potencial zoonótico (Ferreira et al., 2013). 
Existem vários agentes causadores de zoonoses, dentre os principais estão os hemoparasitos que podem usar os cães como hospedeiro definitivo para completar o seu ciclo biológico (Scherer \& Mergener, 2014). Por conta disso, esses animais são susceptíveis a se tornarem fontes de infecção tanto no ambiente domiciliar quanto em áreas públicas, como parques, praias e praças (Nunes et al., 2007), principalmente nas regiões que apresentam baixo desenvolvimento econômico e precárias condições sanitárias (Zanella, 2016).

Dentre as hemoparasitoses que acometem os cães encontra-se a leishmaniose, zoonose que acomete milhares de pessoas e animais todos os anos, resultando em vários problemas de saúde e grandes prejuízos financeiros devido aos custos envolvendo diagnóstico e tratamentos (Ferreira et al., 2013). As manifestações são influenciadas por vários fatores, dentre eles, a espécie, o grau de infestação do parasito, estado nutricional e imunológico do hospedeiro (Tizard, 2014).

Diante do exposto, o presente trabalho tem como objetivo realizar uma revisão sobre os aspectos importantes da leishmaniose visceral canina (LV), como agente etiológico, transmissão e patogenia, sinais clínicos, diagnóstico, tratamento, prevenção e controle.

\section{Características do agente}

A leishmaniose é uma doença causada pelos protozoários do gênero Leishmania sp. que apresenta como hospedeiro principal os cães, cuja transmissão ocorre mediante a picada da fêmea dos mosquitos flebotomíneos (Cortes et al., 2012). A doença é classificada em leishmaniose tegumentar americana e leishmaniose visceral (LV), sendo que esta última é a de maior importância para a saúde pública e a que apresenta a maior ocorrência nos caninos (Feitosa et al., 2000). A leishmaniose visceral é de grande importância para a saúde pública porque é considerada uma zoonose que pode apresentar graves repercussões clínicas (Greene, 2006; Greene \& Vandevelde, 2015).

A LV é causada pelas espécies $L$. infantum, L. donovani e $L$. chagasi, sendo que no território brasileiro a $L$. chagasi é considerada a de maior ocorrência. A $L$. donovani e a $L$. infantum são mais encontradas na Europa, Ásia e África (Camargo \& Barcinski, 2003). Estes protozoários possuem duas morfologias principais, as amastigotas que apresentam a forma redonda ou ovoide, medindo cerca de 1,5 a $2 \mu \mathrm{m}$ de largura e 2,5 a $5 \mu \mathrm{m}$ de comprimento, sendo encontradas principalmente dentro das células do sistema fagocitário mononuclear (SFM) dos hospedeiros definitivos (Greene, 1998), e as promastigotas cujo corpo é alongado e com flagelo, localizadas dentro do tubo digestivo das fêmeas dos flebotomíneos (Jericó et al., 2015).

Os hospedeiros do protozoário são os caninos e o homem (Monteiro, 2011). Todavia, algumas pesquisas apontam os felinos como potenciais hospedeiros acidentais (Can et al., 2016), principalmente em regiões onde a leishmaniose é considerada endêmica com altas taxas de prevalências tanto nos cães quanto nos humanos (Müller et al., 2009). Os roedores, equídeos, caninos e animais silvestres, como o Speothos venaticus (cachorro-vinagre), Cerdocyon thous (cachorro-do-mato), Didelphis albiventris (gambá-de-orelha-branca) e Lycalopex vetulus (raposa-do-mato), podem atuar como reservatórios (Brasil, 2006a). Os vetores ou hospedeiros intermediários são os insetos da espécie Lutzomyia longipalpis, conhecidos vulgarmente como flebotomíneos, tatuquiras, birigui e mosquito-palha (Cortes et al., 2012). Estes mosquitos possuem grande capacidade de adaptação ao clima, por isso estão disseminados por todo território brasileiro, podendo ser encontrado em regiões frias e quentes, úmidas e secas. Em regiões tropicais, a quantidade de flebotomíneos aumenta principalmente durante ou após períodos chuvosos, pois esses períodos de alta umidade favorecem a eclosão das pupas (Almeida et al., 2010). Geralmente esses insetos procuram abrigo em lugares úmidos, com ausência de luminosidade, baixa corrente de ar e com matéria orgânica em processo de decomposição. Dentre os abrigos estão: folhas caídas no solo, tronco de árvores, regiões dentro dos domicílios (Brasil, 2006a).

\section{Transmissão e patogenia}

Nos hospedeiros definitivos, está presente a forma amastigota dentro dos leucócitos e das células do sistema fagocítico mononuclear (SFM). Dentro destas células, o parasito se divide por cissiparidade simples destruindo-as. Já o hospedeiro intermediário, que são as fêmeas dos flebotomíneos, se infectam através da ingestão das formas amastigotas presentes no sangue do vertebrado (Greene \& Vandevelde, 
2015). No intestino do mosquito, as amastigotas se transformam em promastigotas, e por cissiparidade simples se multiplicam rapidamente. Em seguida, os protozoários são inoculados nos vertebrados mediante a picada do flebotomíneo (Fortes, 2004).

Após a inoculação das formas promastigotas nos cães, estas são fagocitadas pelas células do SFM, que são representados principalmente pelos macrófagos. Dentro dos leucócitos, as promastigotas se transformam em amastigotas, as quais se multiplicam rapidamente através de sucessivas divisões binárias. Quando os macrófagos estão densamente parasitados, se rompem liberando várias amastigotas que são fagocitadas novamente por outros macrófagos (Cimerman \& Cimerman, 2005; Neves, 2005). Os protozoários do gênero Leishmania sp. são considerados hemoparasitas, pois podem ser transmitidos através da transfusão sanguínea de animais doadores infectados (Freitas et al., 2006).

\section{Sinais clínicos}

Nos caninos, a leishmaniose pode apresentar a forma clínica assintomática, oligossintomática e sintomática (Brasil, 2006a). Na forma assintomática o animal não apresenta sinais clínicos evidentes da infecção, porém exames sorológicos podem apontar a presença do parasito. A enfermidade é classificada como oligossintomática quando manifesta até dois sinais clínicos e estes são inespecíficos, como pelos opacos, aumento do tamanho dos linfonodos, perda de peso discreta. Neste caso a sorologia poderá apresentar títulos baixos, o que pode resultar na interpretação de um falso-negativo (Brasil, 2006b).

Na forma sintomática várias alterações clínicas podem ser observadas nos cães, como linfadenopatia localizada ou generalizada, polidipsia, poliúria, êmese, polimiosite, poliartrite, neuralgia, febre, onicogrifose, esplenomegalia, ascite, anemia, emagrecimento exagerado, perda de apetite (Tilley et al., 2008; Tilley, 2008), hiperqueratose, alopecia, descamação da pele, eczema nas orelhas e focinho, ulcerações, epistaxe, uveíte, ceratoconjuntivite, blefarite, diarreia, apatia, sonolência, edema nas patas e periostite (Brasil, 2006b). A forma clínica da leishmaniose, está diretamente relacionada ao tipo de resposta celular contra os protozoários. Quando os linfócitos Th1 estão em maior quantidade, ocorre uma resposta celular protetora e o animal não apresenta sinais clínicos. Já quando há predomínio dos linfócitos Th2, ocorre uma resposta imune humoral e o cão apresenta sinais clínicos da infecção (Barbiéri, 2006).

\section{Diagnóstico}

O diagnóstico definitivo da leishmaniose não pode ser baseado apenas nas manifestações clínicas, nos resultados dos exames laboratoriais e na situação epidemiológica da região, pois existem muitas similaridades com outras hemoparasitoses (Jericó et al., 2015). Dentre os testes mais utilizados, estão os moleculares, sorológicos e parasitológicos (Brasil, 2006b).

\section{Exames laboratoriais}

Os principais exames laboratoriais solicitados são o hemograma, perfil bioquímico, proteinograma e urinálise (Ettinger et al., 2002; Greene \& Vandevelde, 2015); porém, os achados clínicos são muito semelhantes aos de outras enfermidades, o que torna estes exames pouco específicos para o diagnóstico (Schimming \& Silva, 2012).

No hemograma podem ser constatados anemia com trombocitopenia, monocitose, linfocitopenia e leucopenia ou leucocitose (Reis et al., 2006). Na maioria das vezes, a anemia é classificada como arregenerativa leve a moderada, normocrômica e normocítica, podendo ser resultante do sequestro de hemácias do baço, hemorragia, eritropoiese diminuída devido a supressão da medula óssea ou deficiência nutricional (Medeiros et al., 2008).

O perfil bioquímico pode apontar hiperproteinemia, aumento das enzimas hepáticas, azotemia e proteinúria (Ettinger et al., 2017). A hiperproteinemia com hipoalbuminemia e hiperglobulinemia, são achados muito frequentes em caninos com leishmaniose devido a proporção reduzida de albumina/globulina. As enzimas hepáticas Alanina aminotransferase (ALT), Fosfatase alcalina (FA) e Gama-glutamiltransferase (GGT), podem sofrer uma elevação discreta ou pronunciada, porém são pouco frequentes nos cães infectados. Na urinálise, uma alta concentração de ureia e creatinina, 
indicativos de azotemia e proteinúria, podem ser observadas nos animais que já apresentam alterações renais devido ao armazenamento de imunocomplexos (Greene \& Vandevelde, 2015).

\section{Diagnóstico parasitológico}

Esta técnica consiste na visualização de formas amastigotas do parasito através do microscópio. $\mathrm{O}$ material biológico a ser analisado, pode ser de esfregaços sanguíneos, citologia aspirativa por agulha fina (CAAF) ou punção aspirativa por agulha fina (PAAF) da medula óssea, linfonodos, baço ou fígado (Teixeira et al., 2010). A aspiração do linfonodo ou medula óssea, é um dos métodos de eleição para o diagnóstico da LV quando a infecção está estabelecida no organismo. Dentre as vantagens estão: elevada especificidade, baixo custo, fácil e rápida execução (Jericó et al., 2015). A desvantagem é que a sensibilidade é baixa, podendo ser influenciada por muitos fatores como: presença de infecções concomitantes, falta de experiência do profissional para a coleta da amostra, tempo transcorrido entre a coleta do material e leitura da lâmina, carga e intensidade do parasitismo (Greene \& Vandevelde, 2015).

\section{Diagnóstico sorológico}

Os testes sorológicos são utilizados devido a elevada quantidade de anticorpos, principalmente a imunoglobulina $\mathrm{G}(\mathrm{IgG})$, produzidas pelos animais infectados. Todavia, não apresentam $100 \%$ de sensibilidade e especificidade. Animais recém infectados ou que estão no período pré-patente, podem apresentar um resultado falso-negativo (Jericó et al., 2015). A presença de doenças concomitantes também pode resultar em falso-positivo, bem como testes realizados em cães filhotes com idade inferior a 5 meses devido a presença dos anticorpos maternos (Larson, 2016). Dentre os exames sorológicos mais utilizados, estão o Teste de aglutinação direta (TAD), RIFI, ELISA (Brasil, 2006a), sendo que no Brasil as técnicas recomendadas para os cães pelo Ministério da Saúde (MS), são estas duas últimas (Greene \& Vandevelde, 2015).

A RIFI em diluição total é considerada o teste padrão para o diagnóstico da leishmaniose visceral, cuja sensibilidade é de aproximadamente $90 \%$ e a especificidade de $80 \%$ (Alves \& Bevilacqua, 2004). Este teste é de fácil execução e baixo custo, porém sua especificidade é afetada por reações cruzadas devido à presença de infecções concomitantes, como Babesia canis, Ehrlichia canis, Neospora caninum e Toxoplasma gondii (Jericó et al., 2015). Em cães com leishmaniose visceral, o teste é considerado reagente, quando são observados títulos de anticorpos iguais ou superiores a diluição de $1 / 40$, mas o teste deve ser repetido dentro de 30 dias para a confirmação do diagnóstico (Brasil, 2006a).

O teste de ELISA é o mais utilizado para o diagnóstico de leishmaniose visceral, dentre suas vantagens estão a maior sensibilidade que o RIFI, possibilita realizar muitos exames em um curto período de tempo, fácil execução e leitura (Souza et al., 2013). A sua sensibilidade permite a detecção de baixos títulos de anticorpos, porém ela diminui em casos assintomáticos e oligo sintomáticos (Gontijo \& Melo, 2004). Outra desvantagem, é a sua menor especificidade em comparação ao RIFI, podendo resultar em maior número de reações cruzadas (Jericó et al., 2015). O resultado do teste é considerado reagente quando o valor da densidade ótica é igual ou acima de três desvios-padrão do cut-off (Brasil, 2006a).

\section{Diagnóstico molecular}

A técnica de PCR, é considerada a mais específica e sensível para o diagnóstico da leishmaniose, pois possibilita identificar o Ácido desoxirribonucleico (DNA) dos protozoários do gênero Leishmania sp., porém apresenta como grande desvantagem o seu custo elevado (Souza et al., 2013). Dentre os materiais biológicos que podem ser analisados, estão o sangue, líquidos corporais, aspirados do baço, medula óssea e linfonodos (Gontijo \& Melo, 2004). A sensibilidade deste exame, é afetada por diversos fatores como metodologia utilizada para extração do DNA e amostras biológicas utilizadas (Nunes et al., 2007). A especificidade e sensibilidade, são maiores para amostras de materiais obtidas de linfonodos, baço, medula óssea e pele (Greene \& Vandevelde, 2015).

\section{Diagnóstico diferencial}

Existem muitas doenças que apresentam similaridade nos sinais clínicos apresentados pelos animais, dentre as principais estão: demomicose e piodermite bacteriana para alopecia e descamação de pele; 
infecção fúngica e Lúpus Eritematoso Sistémico para ulcerações cutâneas. Quando os sinais clínicos são sistêmicos, deve suspeitar de erliquiose, babesiose e hepatozoonose (Pessoa-e-Silva et al., 2019).

\section{Tratamento}

Pela Nota Técnica no 11/2016, assinada pelo Ministério da Agricultura Pecuária e Abastecimento (MAPA) e pelo MS, foi aprovado o registro do medicamento MILTEFORAN ${ }^{\mathrm{TM}}$, como único produto preconizado para o tratamento da leishmaniose visceral de cães no Brasil (Brasil, 2016). Porém, outros medicamentos são utilizados para auxiliar no tratamento da leishmaniose, dentre eles o alopurinol e a domperidona (Greene \& Vandevelde, 2015). A miltefosina é o princípio ativo do MILTEFORANTM, o qual apresenta efeito tóxico sobre os protozoários da leishmaniose. Quanto a sua posologia, deve ser administrada pela via oral na dose de $2 \mathrm{Mg} / \mathrm{kg} /$ dia durante 4 semanas (Greene \& Vandevelde, 2015).

O alopurinol interrompe a síntese proteica do parasito, resultando na inibição da sua multiplicação e na morte (Jericó et al., 2015). Este fármaco é comumente utilizado em associação a outros medicamentos, pois parece exercer efeitos leishmaniostáticos e é pouco tóxico e de baixo custo (Jericó et al., 2015). A sua administração é por via oral na dose de $10 \mathrm{Mg} / \mathrm{Kg}$ duas vezes ao dia (BID), e resulta em melhora clínica dentro de 4 semanas. Produz poucos efeitos colaterais, dentre eles está a hiperxantinúria que pode resultar em urolitíases (Greene \& Vandevelde, 2015).

A domperidona não apresenta um efeito direto sobre os hemoprotozoários, porém é utilizado em associação com outros medicamentos, devido ao seu efeito imunomodulador ajudar na redução das manifestações clínicas de animais infectados com leishmaniose (Gómez-Ochoa et al., 2009). Antes da realização do tratamento da leishmaniose, é necessário identificar qual é o grau de estadiamento da doença no animal a fim de prescrever a melhor protocolo terapêutico possível (Greene \& Vandevelde, 2015).

\section{Prevenção e controle}

A eutanásia de cães sororreagentes para leishmaniose, foi recomendada como medida de controle pela Organização Mundial da Saúde (OMS) e pela Organização Pan-Americana da Saúde (OPAS) (Jericó et al., 2015); porém, estudos afirmaram que esta metodologia é ineficaz pois a transmissão ocorre através dos vetores flebotomíneos, e existem diversas espécies de animais selvagens que podem atuar como reservatórios (Greene \& Vandevelde, 2015).

Para diminuir os riscos de transmissão, algumas medidas de proteção individual podem ser adotadas, como a utilização de mosquiteiro, tela em janelas e portas, repelentes e inseticidas (Brasil, 2014). Além disso, também é recomendado realizar levantamento sorológico dos animais nas áreas endêmicas, educação sanitária na população e limpeza de terrenos baldios (Fortes, 2004).

Para cães, coleiras impregnadas com Deltametrina a 4\% em condições experimentais, demonstraram boa eficácia como repelente de flebotomíneos (E. B. Alves et al., 2018). Também podem ser utilizados repelentes naturais a base de citronela e extrato de neem, e inseticidas tópicos na forma de spray a base de permetrina (Jericó et al., 2015). Existe a vacina Leish-Tec ${ }^{\circledR}$, a qual é a única licenciada pelas autoridades da saúde pública do Brasil, para a venda e administração por médicos veterinários (Matias et al., 2020). Conforme pesquisas realizadas por Fernandes et al. (2008), essa vacina foi capaz de induzir imunidade contra a infecção induzida por alta dose intravenosa de L. chagasi em cães da raça beagles. Além disso, também foi testada em populações heterogêneas de cães, e apontou ser segura e bem tolerada pelos animais (Testasicca et al., 2014).

\section{Conclusão}

A leishmaniose visceral canina possui grande importância no âmbito da saúde pública e animal, por isso há necessidade de realizar estudos epidemiológicos com o objetivo de elaborar planos mais eficazes para o seu controle. Para isso, faz-se necessário conhecimento do agente etiológico e sua respectiva patogenia, afim de garantir eficácia no diagnóstico e consequentemente determinar um tratamento adequado.

\section{Referências}

Almeida, P. S., Minzão, E. R., Minzão, L. D., Silva, S. R., Ferreira, A. D., Faccenda, O., \& Andrade 
Filho, J. D. (2010). Aspectos ecológicos de flebotomíneos (Diptera: Psychodidae) em área urbana do município de Ponta Porã, Estado de Mato Grosso do Sul. Revista Da Sociedade Brasileira de Medicina Tropical, 43(6), 723-727. DOI: https://doi.org/10.1590/s0037-86822010000600025

Alves, E. B., Figueiredo, F. B., Rocha, M. F., \& Werneck, G. L. (2018). Dificuldades operacionais no uso de coleiras caninas impregnadas com inseticida para o controle da leishmaniose visceral, Montes Claros, MG, 2012. Epidemiologia e Serviços de Saúde, 27, e2017469. DOI: https://doi.org/10.5123/S1679-49742018000400001

Alves, W. A., \& Bevilacqua, P. D. (2004). Reflexões sobre a qualidade do diagnóstico da leishmaniose visceral canina em inquéritos epidemiológicos: o caso da epidemia de Belo Horizonte, Minas Gerais, Brasil, 1993-1997. Cadernos de Saúde Pública, 20, 259-265. DOI: https://doi.org/10.1590/s0102$\underline{311 \times 2004000100043}$

Barbiéri, C. L. (2006). Immunology of canine leishmaniasis. Parasite Immunology, 28(7), 329-337. DOI: https://doi.org/10.1111/j.1365-3024.2006.00840.x

Brasil. (2006a). Ministério da Saúde. Secretaria de Vigilância em Saúde. Departamento de Vigilância Epidemiológica. Manual de vigilância e controle da leishmaniose visceral / Ministério da Saúde, Secretaria de Vigilância em Saúde, Departamento de Vigilância Epidemiológica. Editora do Ministério da Saúde, Brasília, Brasil.

Brasil. (2006b). Superintendência de Controle de Endemias do Estado de São Paulo -SUCEN. Relatório Leishmaniose Visceral.

Brasil. (2014). Ministério da Saúde. Secretaria de Vigilância em Saúde. Departamento de Vigilância Epidemiológica. Manual de Vigilância e Controle da Leishmaniose Visceral. Editora do Ministério da Saúde, Brasília, Brasil.

Brasil. (2016). Ministério da Agricultura Pecuária e Abastecimento. Nota Técnica $N^{o}$ 11/2016/CPV/DFIP/SDA/GM/MAPA.

Caldart, E. T., Constantino, C., Pasquali, A. K. S., Benitez, A. N., Hamada, F. N., Dias, R. C. F., RoratoNascimento, A. M., Marana, E. R. M., Navarro, I. T., \& Mascarenhas, N. M. F. (2015). Zoonosis in dogs and cats attended by the Birth Control Project: Toxoplasma gondii, Leishmania spp. and Leptospira spp., serodiagnosis and epidemiology. Semina: Ciências Agrárias, 36(1), 253-265. DOI: https://doi.org/10.5433/1679-0359.2015v36n1p253

Camargo, L. M. A., \& Barcinski, M. A. (2003). Leishmanioses, feridas bravas e kalazar. Ciência e Cultura, 55(1), 34-37.

Can, H., Döşkaya, M., Özdemir, H. G., Şahar, E. A., Karakavuk, M., Pektaş, B., Karakuş, M., Töz, S., Caner, A., \& Döşkaya, A. D. (2016). Seroprevalence of Leishmania infection and molecular detection of Leishmania tropica and Leishmania infantum in stray cats of İzmir, Turkey. Experimental Parasitology, 167, 109-114. DOI: https://doi.org/10.1016/j.exppara.2016.05.011

Cimerman, B., \& Cimerman, S. (2005). Parasitologia humana e seus fundamentos gerais. In Parasitologia humana e seus fundamentos gerais (p. 390). Atheneu Editora.

Cortes, S., Vaz, Y., Neves, R., Maia, C., Cardoso, L., \& Campino, L. (2012). Risk factors for canine leishmaniasis in an endemic Mediterranean region. Veterinary Parasitology, 189(2-4), 189-196. DOI: https://doi.org/10.1016/j.vetpar.2012.04.028

Ettinger, S. J., Fedlman, E. C., \& Taibo, R. A. (2002). Tratado de medicina interna veterinaria: enfermedades del perro y el gato. Manole.

Ettinger, S. J., Feldman, E. C., \& Cote, E. (2017). Textbook of Veterinary Internal Medicine-eBook. Elsevier Health Sciences.

Feitosa, M. M., Ikeda, F. A., Luvizotto, M. C. R., \& Perri, S. H. V. (2000). Clinical aspects of dogs with visceral leishmaniasis from Araçatuba-São Paulo State (Brazil). Clínica Veterinária, 5(28), 36-44.

Fernandes, A. P., Costa, M. M. S., Coelho, E. A. F., Michalick, M. S. M., Freitas, E., Melo, M. N., Tafuri, W. L., Resende, D. M., Hermont, V., \& Abrantes, C. F. (2008). Protective immunity against challenge with Leishmania (Leishmania) chagasi in beagle dogs vaccinated with recombinant A2 protein. Vaccine, 26(46), 5888-5895. DOI: https://doi.org/10.1016/j.vaccine.2008.05.095

Ferreira, F. P., Dias, R. C. F., Martins, T. A., Constantino, C., Pasquali, A. K. S., Vidotto, O., Freire, R. 
L., \& Navarro, I. T. (2013). Frequência de parasitas gastrointestinais em cães e gatos do município de Londrina, PR, com enfoque em saúde pública. Semina: Ciências Agrárias, 34(6), 3851-3858. DOI: https://doi.org/10.5433/1679-0359.2013v34n6supl2p3851

Fortes, E. (2004). Parasitologia veterinária (4th ed.). Editora ícone.

Freitas, E., Melo, M. N., Costa-Val, A. P., \& Michalick, M. S. M. (2006). Transmission of Leishmania infantum via blood transfusion in dogs: potential for infection and importance of clinical factors. Veterinary Parasitology, 137(1-2), 159-167. DOI: https://doi.org/10.1016/j.vetpar.2005.12.011

Gómez-Ochoa, P., Castillo, J. A., Gascón, M., Zarate, J. J., Alvarez, F., \& Couto, C. G. (2009). Use of domperidone in the treatment of canine visceral leishmaniasis: a clinical trial. The Veterinary Journal, 179(2), 259-263. DOI: https://doi.org/10.1016/j.tvjl.2007.09.014

Gontijo, C. M. F., \& Melo, M. N. (2004). Leishmaniose visceral no Brasil: quadro atual, desfios e perspectivas. Revista Brasileira de Epidemiologia, 7(3), 338-349. DOI: https://doi.org/10.1590/s1415-790x2004000300011

Greene, C. E. (1998). Canine distemper. In C. E. Greene (Ed.), Infections diseases of the dog and the cat (p. 820). Editora Interamericana.

Greene, C. E. (2006). Fatores ambientais de doenças infecciosas. In E. D. Ibid (Ed.), Doenças infecciosas em cães e gatos. Elsivier.

Greene, C. E., \& Vandevelde, M. (2015). Cinomose. In C. E. Greene (Ed.), Doenças infecciosas em cães e gatos. Guanabara Koogan.

Hoffmann, A. R., Navarro, I. T., Camargo Junior, V. E., Caldart, E. T., Breganó, R. M., \& Pereira, P. M. (2012). Leishmania amazonensis em cão com quadro clínico de leishmaniose visceral no Estado do Paraná, Brasil-relato de caso. Semina: Ciências Agrárias, 33(2), 3265-3270. DOI: https://doi.org/10.5433/1679-0359.2012v33supl2p3265

Jericó, M. M., Kogika, M. M., \& Andrade Neto, J. P. (2015). Tratado de medicina interna de cães e gatos. Guanabara Koogan.

Larson, C. E. (2016). Tricologia. In C. E. Larson \& R. Lucas (Eds.), Tratado de medicina externa: dermatologia veterinária. Interbook.

Matias, E. P., Santos, F. F., Guimarães, J. P., \& Chucri, T. M. (2020). Efeitos adversos da vacina LEISHTEC® em cães soronegativos para Leishmaniose Visceral. Brazilian Journal of Development, 6(7), 53019-53028. DOI: https://doi.org/10.34117/bjdv6n7-810

McNicholas, J., Gilbey, A., Rennie, A., Ahmedzai, S., Dono, J.-A., \& Ormerod, E. (2005). Pet ownership and human health: a brief review of evidence and issues. British Medical Journal, 331(7527), 1252-1254. DOI: https://doi.org/10.1136/bmj.331.7527.1252

Medeiros, C. M. O., Melo, A. G. C., Lima, A. K. F., Silva, I. N. G., Oliveira, L. C., \& Silva, M. C. (2008). Perfil hematológico de cães com leishmaniose visceral nomunicípio de Fortaleza, Ceará. Ciência Animal, 18(1), 43-50.

Monteiro, S. G. (2011). Parasitologia na medicina veterinária (Vol. 1). Roca.

Müller, D. C. M., Pippi, N. L., Basso, P. C., Olsson, D. C., Santos Junior, E. B., \& Guerra, A. C. O. (2009). Técnicas e sítios de coleta de medula óssea em cães e gatos. Ciência Rural, 39(7), 2243 2251. DOI: https://doi.org/10.1590/s0103-84782009005000153

Neves, D. P. (2005). Parasitologia humana. Editora Atheneu.

Nunes, C. M., Dias, A. K. K., Gottardi, F. P., Paula, H. B., Azevedo, M. A. A., Lima, V. M. F., \& Garcia, J. F. (2007). Avaliação da reação em cadeia pela polimerase para diagnóstico da leishmaniose visceral em sangue de cães. Revista Brasileira de Parasitologia Veterinária, 16(2), 67-71.

Pessoa-e-Silva, R., Vaitkevicius-Antão, V., Andrade, T. A. S., Silva, A. C. O., Oliveira, G. A., TrajanoSilva, L. A. M., Nakasone, E. K. N., \& Paiva-Cavalcanti, M. (2019). The diagnosis of canine visceral leishmaniasis in Brazil: Confronting old problems. Experimental Parasitology, 199, 9-16. DOI: https://doi.org/10.1016/j.exppara.2019.02.012

Reis, A. B., Martins-Filho, O. A., Teixeira-Carvalho, A., Carvalho, M. G., Mayrink, W., França-Silva, J. C., Giunchetti, R. C., Genaro, O., \& Corrêa-Oliveira, R. (2006). Parasite density and impaired 
biochemical/hematological status are associated with severe clinical aspects of canine visceral leishmaniasis. Research in Veterinary Science, 81(1), 68-75. DOI: https://doi.org/10.1016/j.rvsc.2005.09.011

Scherer, M., \& Mergener, M. (2014). Prevalência de hemocitozoários em caninos de municípios do Vale do Taquari com foco em Lajeado-RS. Revista Destaques Acadêmicos, 6(3), 206-212.

Schimming, B. C., \& Silva, J. R. C. P. (2012). Leishmaniose visceral canina - Revisão de literatura. Revista Científica Eletrônica de Medicina Veterinária, 10(18), 1-5. DOI: https://doi.org/10.5892/ruvrd.v16i1.3383

Souza, Y. C. P., Carvalho, A. F. S., Carvalho, L. A. R., \& Mansur, V. F. R. (2013). Testes diagnósticos para leishmaniose visceral: atualidade e perspectivas. Revista Científica Eletrônica de Medicina Veterinária, 11(21), 1-16.

Teixeira, L. V, Lopes, S. T. A., Martins, D. B., França, R. T., \& Fighera, R. A. (2010). Punção aspirativa por agulha fina como método de coleta de material para a histopatologia no osteossarcoma canino. Pesquisa Veterinária Brasileira, 30(2), 132-138. DOI: https://doi.org/10.1590/s0100$\underline{736 \times 2010000200008}$

Testasicca, M. C. S., Santos, M. S., Machado, L. M., Serufo, A. V., Doro, D., Avelar, D., Tibúrcio, A. M. L., Abrantes, C. F., Machado-Coelho, G. L. L., \& Grimaldi Junior, G. (2014). Antibody responses induced by Leish-Tec $\AA$, an A2-based vaccine for visceral leishmaniasis, in a heterogeneous canine population. Veterinary Parasitology, 204(3-4), 169-176. DOI: https://doi.org/10.1016/j.vetpar.2014.04.025

Tilley, Larry Patrick, Smith, J. R., \& Francis, W. K. (2008). Consulta veterinária em 5 minutos: Espécies canina e felina. Editora Manole.

Tilley, Lawrence P. (2008). Manual of canine and feline cardiology. Elsevier Health Sciences.

Tizard, I. R. (2014). Imunologia Veterinária: introdução. Editora Roca.

Zanella, J. R. C. (2016). Frequência de parasitas gastrointestinais em cães e gatos do município de Londrina, PR, com enfoque em saúde pública. Pesquisa Agropecuária Brasileira, 51, 510-519

Histórico do artigo:

Recebido: 14 de agosto de 2020. Aprovado: 12 de outubro de 2020 .

Disponível online: 26 de janeiro de 2021.
Licenciamento: Este artigo é publicado na modalidade Acesso Aberto sob a licença Creative Commons Atribuição 4.0 (CC-BY 4.0), a qual permite uso irrestrito, distribuição, reprodução em qualquer meio, desde que o autor e a fonte sejam devidamente creditados. 